\title{
Pure Spin Current and Magnon Chemical Potential in a Nonequilibrium Magnetic Insulator
}

\author{
Kevin S. Olsson $\odot,{ }^{1}$ Kyongmo An, ${ }^{2}$ Gregory A. Fiete, ${ }^{1,3,4}$ Jianshi Zhou, ${ }^{5,6}$ Li Shi ${ }^{5,6}$ and Xiaoqin Li ${ }^{1,5, *}$ \\ ${ }^{1}$ Department of Physics, Center of Complex Quantum Systems, \\ The University of Texas at Austin, Austin, Texas 78712, USA \\ ${ }^{2}$ Laboratory of Nanoscale Magnetic Materials and Magnonics, Institute of Materials, School of \\ Engineering, École Polytechnique Fédérale de Lausanne (EPFL), 1015 Lausanne, Switzerland \\ ${ }^{3}$ Department of Physics, Northeastern University, Boston, Massachusetts 02115, USA \\ ${ }^{4}$ Department of Physics, Massachusetts Institute of Technology, Cambridge, Massachusetts 02139, USA \\ ${ }^{5}$ Texas Materials Institute, The University of Texas at Austin, Austin, Texas 78712, USA \\ ${ }^{6}$ Department of Mechanical Engineering, The University of Texas at Austin, Austin, Texas 78712, USA
}

(Received 2 June 2019; revised manuscript received 18 February 2020; accepted 27 February 2020; published 6 May 2020)

\begin{abstract}
Nonequilibrium phenomena are ubiquitous in nature and in a wide range of systems, including cold atomic gases and solid-state materials. While these phenomena are challenging to describe both theoretically and experimentally, they are essential for the fundamental understanding of many-body systems and practical devices. In the context of spintronics, when a magnetic insulator (MI) is subjected to a thermal gradient, a pure spin current is generated in the form of magnons without the presence and dissipation of a charge current-attractive for reducing energy consumption and central to the emerging field of spin caloritronics. However, the experimental methods for directly quantifying a spin current in insulators and for probing local phonon-magnon nonequilibrium and the associated magnon chemical potential are largely missing. Here, we apply a heating laser to generate a thermal gradient in the MI yttrium iron garnet (YIG), $\mathrm{Y}_{3} \mathrm{Fe}_{5} \mathrm{O}_{12}$, and evaluate two components of the spin current, driven by temperature and chemical potential gradients, respectively. The experimental method and theory approach for evaluating quasiparticle chemical potential can be applied for analogous phenomena in other many-body systems.
\end{abstract}

DOI: 10.1103/PhysRevX.10.021029

Subject Areas: Condensed Matter Physics, Magnetism, Spintronics

\section{INTRODUCTION}

Magnetic insulators (MIs) provide a unique model system for exploring nonequilibrium phenomena and developing spintronic applications [1-3]. What is appealing to both fields is the fact that properties of MIs are determined by only two subsystems, i.e., two types of collective excitations: phonons and magnons, leading to longer energy and spin relaxation times or lengths than those in metallic systems. In particular, thermally driven nonequilibria and the interplay between these phonons and magnons result in a broad range of newly discovered spin caloritronic phenomena, such as the spin Seebeck effect [4-6]. Understanding these phenomena requires simultaneous descriptions of nonequilibrium energy (heat) and

\footnotetext{
* Corresponding author. elaineli@physics.utexas.edu

Published by the American Physical Society under the terms of the Creative Commons Attribution 4.0 International license. Further distribution of this work must maintain attribution to the author(s) and the published article's title, journal citation, and DOI.
}

spin transport [7-12]. Theoretical and experimental advances are both necessary to address the outstanding challenges in this emerging frontier of spintronics and nonequilibrium systems, in general.

In this paper, we measure thermally generated spin current in YIG and quantitatively evaluate its two components driven by temperature and magnon chemical potential gradients. More specifically, a heating laser is used to introduce a large temperature gradient [9]. Along this gradient, micro Brillouin light scattering (BLS) was used to measure the local lattice temperature and nonequilibrium magnon density, from which the spatially varying magnon chemical potential is explicitly determined. Based on the magnon temperature and chemical potential profiles, we quantify two components of the spin current and calculate the magnon nonequilibrium spectral density. This new method for measuring spin current in MIs without any interfacial effects, as well as quantitative evaluation of the magnon chemical potential and nonequilibrium magnon distribution, will stimulate further development of spin caloritronic theories and devices.

We first describe the basic concepts involved in understanding magnon transport in a nonequilibrium system. 

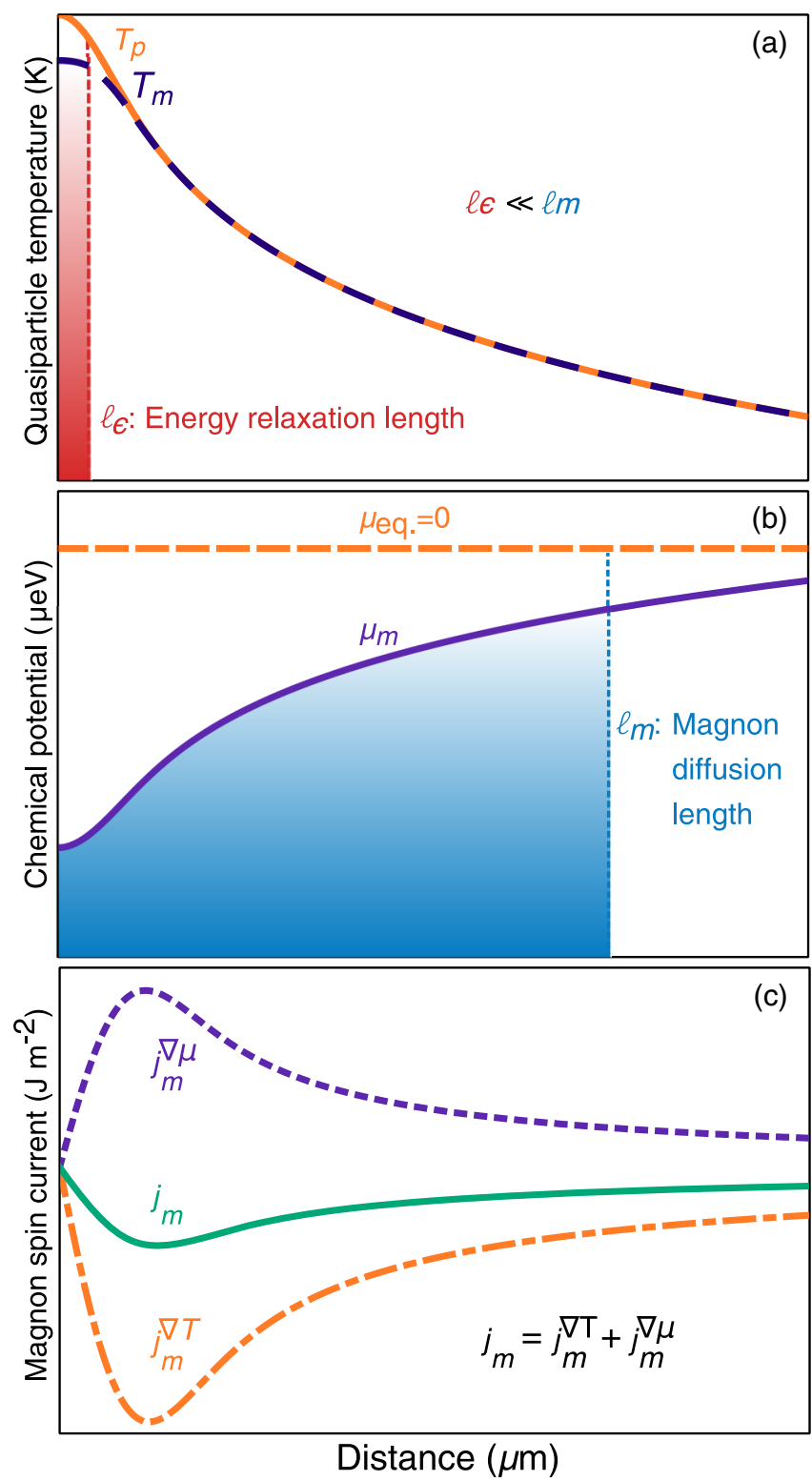

FIG. 1. Diagram of magnons in thermal nonequilibrium for a magnetic insulator under a temperature gradient $\nabla T$. There are two relevant relaxation lengths: (a) The energy relaxation length $l_{\epsilon}$, describes the length scale required for the magnon and phonon temperatures, $T_{m}$ and $T_{p}$, respectively, to equilibrate. (b) A magnon chemical potential $\mu_{m}$ parameterizes the nonequilbrium magnon density such that $\mu_{m}$ approaches the equilibrium value 0 over the magnon diffusion length $l_{m}$. (c) The magnon spin current $j_{m}$ is comprised of two components, $j_{m}^{\nabla T}$ and $j_{m}^{\nabla \mu}$, which, respectively, result from the gradients of the magnon temperature and chemical potential. For temperature and chemical potential gradients with opposite signs, as in panels (a) and (b), the corresponding spin currents have the opposite signs as well.

The energy exchange between magnons and phonons is described by the local phonon and magnon temperatures. Following the magnon-phonon energy relaxation length $\ell_{\epsilon}$, phonon and magnon temperatures equilibrate, as illustrated in Fig. 1(a). This length has been initially predicted to be on the order of about $1 \mathrm{~nm} \mathrm{[10]} \mathrm{and} \mathrm{found} \mathrm{experimentally} \mathrm{to} \mathrm{be}$ about $250 \mathrm{~nm}$ for YIG $[12,13]$. Spin transport in the MI is characterized by the magnon spin relaxation length $\ell_{m}$, illustrated in Fig. 1(b), describing the length scale of angular momentum (spin) relaxation. Previous measurements of YIG have found $\ell_{m}$ in the range of 3-10 $\mu \mathrm{m}$ $[8,9,11]$, much larger than $\ell_{\epsilon}$.

On length scales shorter than $\ell_{m}$ but longer than $\ell_{\epsilon}$, the magnon number is approximately conserved and the magnon population can be driven to a value different from that solely determined by the magnon temperature. The difference in the magnon population is described by introducing another parameter, specifically, a magnon chemical potential $\mu_{m}$ [10-12,14]. Gradients in both magnon temperature and chemical potential result in a magnon spin current [Fig. 1(c)], carried by magnons with an energy distribution characterized by the nonequilibrium spectral density. Spin currents in MIs characterized by transport measurements have several limitations. First, spin current in insulators is not directly measured. Instead, the inverse spin Hall effect in an adjacent heavy metal layer is used to measure and estimate the spin current in the MI and is thus subject to properties of the metal layer and to sample-preparation-dependent interfacial effects. Second, such measurements cannot distinguish between spin currents driven by different mechanisms due to the difficulty of evaluating the magnon chemical potential. While the magnon chemical potential has been experimentally evaluated in the context of microwave pumping, magnetic aftereffect, and spin current injection [15-18], a thermal gradient driven, spatially dependent, magnon chemical potential most relevant to spin caloritronic applications has yet to appear in the literature. We report such a measurement here.

\section{EXPERIMENT}

The experimental setup is illustrated in Fig. 2(a). A 671-nm heating laser, with a spot size of $2.3 \mu \mathrm{m}$ in diameter and power of $22.7 \mathrm{~mW}$, was used to generate a thermal gradient of about $10^{4} \mathrm{~K} / \mathrm{mm}$ in a bulk YIG single crystal (Sec. III A). BLS spectra of phonons and magnons were collected in a backscattering geometry using a 532-nm probe laser, with a spot size of $0.8 \mu \mathrm{m}$ in diameter and a power of $8 \mathrm{~mW}$. Spectra collected at different probe positions relative to the heated spot exhibit a systematic change due to the corresponding changes in the effective local temperature and magnon density $[9,19,20]$. Examples of the phonon [Fig. 2(b)] and magnon [Fig. 2(c)] spectra were taken at two positions - near the center and the edge of the heated spot. Central frequencies were extracted from the fits to squared Lorentzian functions shown as the solid curves in Figs. 2(b) and 2(c). The wave vectors of the phonon and magnon modes are the same and are both determined by the geometry, refractive index, and incident 

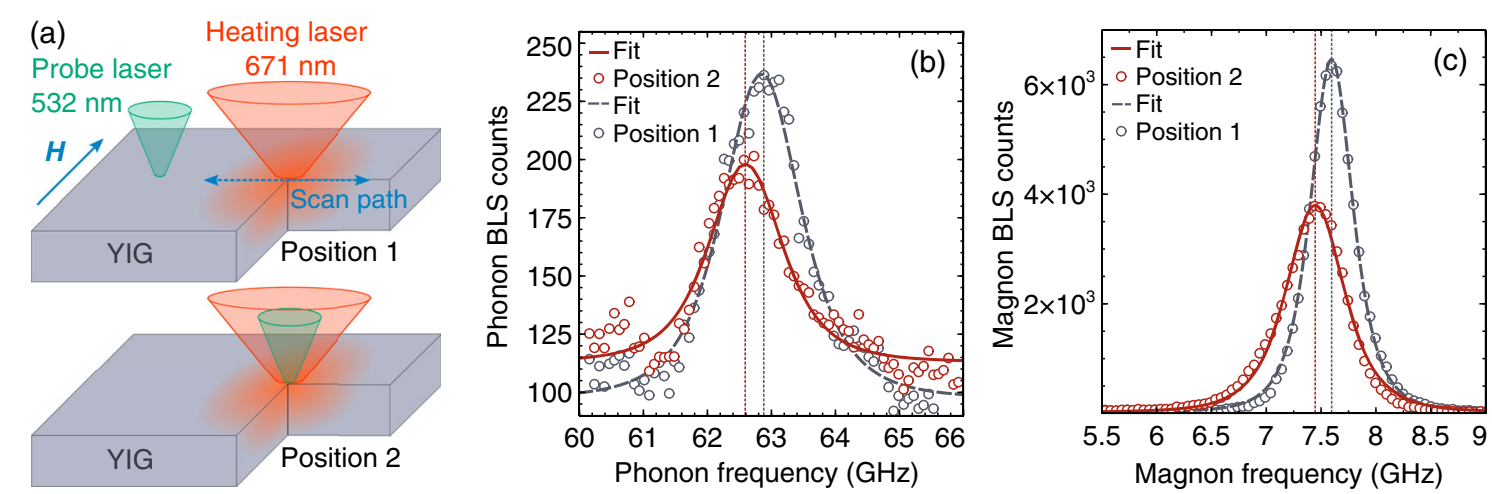

FIG. 2. Experimental setup and BLS spectra. (a) A 671-nm laser was applied to a bulk YIG single crystal in order to generate a large thermal gradient over a micron length scale. The BLS spectra were collected as the 532-nm probe laser was scanned across the thermal gradient. The measured magnons and phonons propogate along the direction of the incident wave vector of the probing laser. Example spectra for acoustic phonons (b) and magnons (c) at the edge (position 1) and center (position 2) of the temperature gradient. Squared Lorentzian fits (curves) allow for the extraction of the central phonon and magnon frequencies (vertical, dotted lines) along the scan path. In panel (c), the counts for position 2 have been multiplied by 2 for better comparison to position 1 .

wave vector (Sec. III B). Here, the phonon or magnon wave vector is $5.53 \times 10^{7} \mathrm{~m}^{-1}$, corresponding to a wavelength of $114 \mathrm{~nm}$. The measurements are most sensitive to those magnons and phonons propagating along the direction of the probe laser wave vector. We note the measured BLS spectra represent the spectra averaged over the intensity profile of the probing laser (see Supplemental Material [21]).

\section{MATERIALS AND METHODS}

\section{A. YIG sample}

The YIG single crystal was grown via the travelingsolvent floating-zone method by using an infrared-heated image furnace [22]. The sample measured was approximately $4 \mathrm{~mm} \times 2 \mathrm{~mm} \times 0.4 \mathrm{~mm}$, with the [110] crystal axis oriented normal to the surface. An external magnetic field of $78 \mathrm{mT}$ was applied along the sample plane, perpendicular to the [110] axis, to saturate the magnetization of the sample [23].

\section{B. Phonon and magnon BLS spectra}

A Sandercock-type multipass tandem Fabry-Perot interferometer was used to collect both the magnon and acoustic phonon spectra. A mirror spacing of $6.00 \pm 0.05 \mathrm{~mm}$, corresponding to a free spectral range (FSR) of $25.0 \pm$ $0.2 \mathrm{GHz}$ for the first Fabry-Perot cavity, was used for all spectra collection. In collecting the magnon spectra, data were collected over $22 \%-35 \%$ of the FSR, using 600 channels per FSR on the multichannel analyzer (MCA). Phonon spectra were collected over $244.5 \%-265.0 \%$ of the FSR, with 200 channels per FSR on the MCA. The time needed to sweep over the frequency range of one spectrum is determined by the highest frequency in the region of intereste and the channels per FSR. In order to have the interferometer stay in alignment for the phonon spectra, the channels per FSR had to be reduced as the spectra were being swept over a larger frequency range. We also followed a particular measurement sequence to monitor a systematic shift in frequency due to thermal drift over time. We took phonon and magnon spectra under a particular heating condition and immediately took another set of data with the heating laser off.

The momentum transfer of the light in backscattering geometry determines the wave vector $q$ of the probed phonons and magnons, such that $q=2 n k_{0}$ [9]. For normal incidence and a probe wavelength $532 \mathrm{~nm}$, the incident light wave vector is $k_{0}=2 \pi / \lambda=1 \times 10^{7} \mathrm{~m}^{-1}$, and the YIG refractive index is $n=2.34$, which together yield $q=5.53 \times 10^{7} \mathrm{~m}^{-1}$. However, as the objective lens used to focus the light has a high numerical aperture $(\mathrm{NA}=0.7)$, the incident light is not confined to a single wave vector. Thus, the measured BLS spectra are centered around $q=5.53 \times 10^{7} \mathrm{~m}^{-1}$, with a $0.25 \times 10^{7} \mathrm{~m}^{-1}$ wave-vector spread contributing to the broadening of the linewidth.

The acoustic phonon frequency is given by

$$
\omega_{p}(k)=v k,
$$

where $v$ is the group velocity of the probed phonon. In YIG, this velocity is $7.3 \times 10^{3} \mathrm{~ms}^{-1}$ for longitudinal phonons along the [110] direction. This result predicts a phonon frequency of $63.4 \mathrm{GHz}$, in agreement with the central frequency observed in the BLS phonon spectra [Fig. 2(b)]. The frequency of the magnon is given by the following dispersion relation [24]:

$$
\omega_{k}=\gamma \sqrt{\begin{array}{c}
\left(B_{\mathrm{eff}}+D k^{2}\right) \\
\times\left(B_{\mathrm{eff}}+D k^{2}+\mu_{0} M_{s}(T) \sin ^{2} \psi\right)
\end{array}},
$$

where $\gamma$ is the gyromagnetic ratio of $2 \pi \times 2.80 \mathrm{MHz} \mathrm{G}^{-1}$, $B_{\text {eff }}$ is the effective magnetic field, $D$ is the exchange 
stiffness constant of $5.4 \times 10^{-17} \mathrm{Tm}^{2}, \mu_{0}$ is the permeability of free space, $M_{s}(T)$ is the temperature-dependent saturation magnetization (see Supplemental Material [21]), and $\psi$ is the angle between $k$ and the magnetization direction $[9,20]$. The effective field includes the external magnetic field of $78 \mathrm{mT}$, the anisotropy field of $1 \mathrm{mT}$, and the demagnetization field $\mu_{0} N M_{s}(T)$, where the calculated demagnetization factor is $N=0.08$ [9]. These parameters yield a predicted magnon frequency of $7.6 \mathrm{GHz}$, in agreement with the BLS magnon spectra [Fig. 2(c)].

\section{Equilibrium frequency-to-temperature calibration}

The phonon and magnon thermometry methods based on BLS spectra are essential to the analysis $[9,19,20]$. Calibrations are performed by uniformly heating the sample and monitoring the BLS spectra as a function of temperature, such that changes in the spectra result from the changing equilibrium lattice temperature. This procedure yields two temperature-dependent frequency functions, $f_{p}(T)$ and $f_{m}(T)$, for the phonons and magnons, respectively.

From $f_{p}(T)$, the inverse function $T\left(f_{p}\right)$ is calculated and used for measuring the increase of phonon temperature, $\Delta T_{p}$, from the phonon frequency deviations $\Delta f_{p}$, $\Delta T_{p}=T\left(\Delta f_{p}\right)$. The function $f_{m}(T)$ gives the frequency for magnons at temperature $T$. Thus, using this magnon calibration function with the simulated increase of magnon temperature, $\Delta T_{m}$, we obtain the expected equilibrium magnon frequency change, $\Delta f_{m}^{\mathrm{cal}}=\Delta f_{m}\left(\Delta T_{m}\right)$. See Supplemental Material [21] for more details on the calibration and heating effects from the probe laser.

\section{RESULTS AND DISCUSSION}

\section{A. Quantifying magnon nonequilibrium}

At local equilibrium, the magnon and phonon temperatures are equal, and the magnon chemical potential is zero. Evaluating the magnon nonequilibrium begins with determining the phonon temperature. The spatially dependent phonon frequencies extracted from the BLS spectra are converted to the effective local phonon temperatures following a calibration procedure. The increase of phonon temperature due to the heating laser $\Delta T_{p}$ can be extracted from the phonon frequency deviation $\Delta f_{p}=f_{p}^{\text {on }}-f_{p}^{\text {off }}$, which is the difference in phonon frequencies measured for the on and off heating laser conditions. This difference in frequencies removes the effect due to heating from the probe laser, which is present for all measurements. Figure 3(a) shows $\Delta f_{p}$ measured across the laser-heated region, with the associated local increase of phonon temperature shown in Fig. 3(b). As expected, the increase in phonon temperature is the highest at the center of the heating laser and decreases with the distances further from the center. The empirical fitting
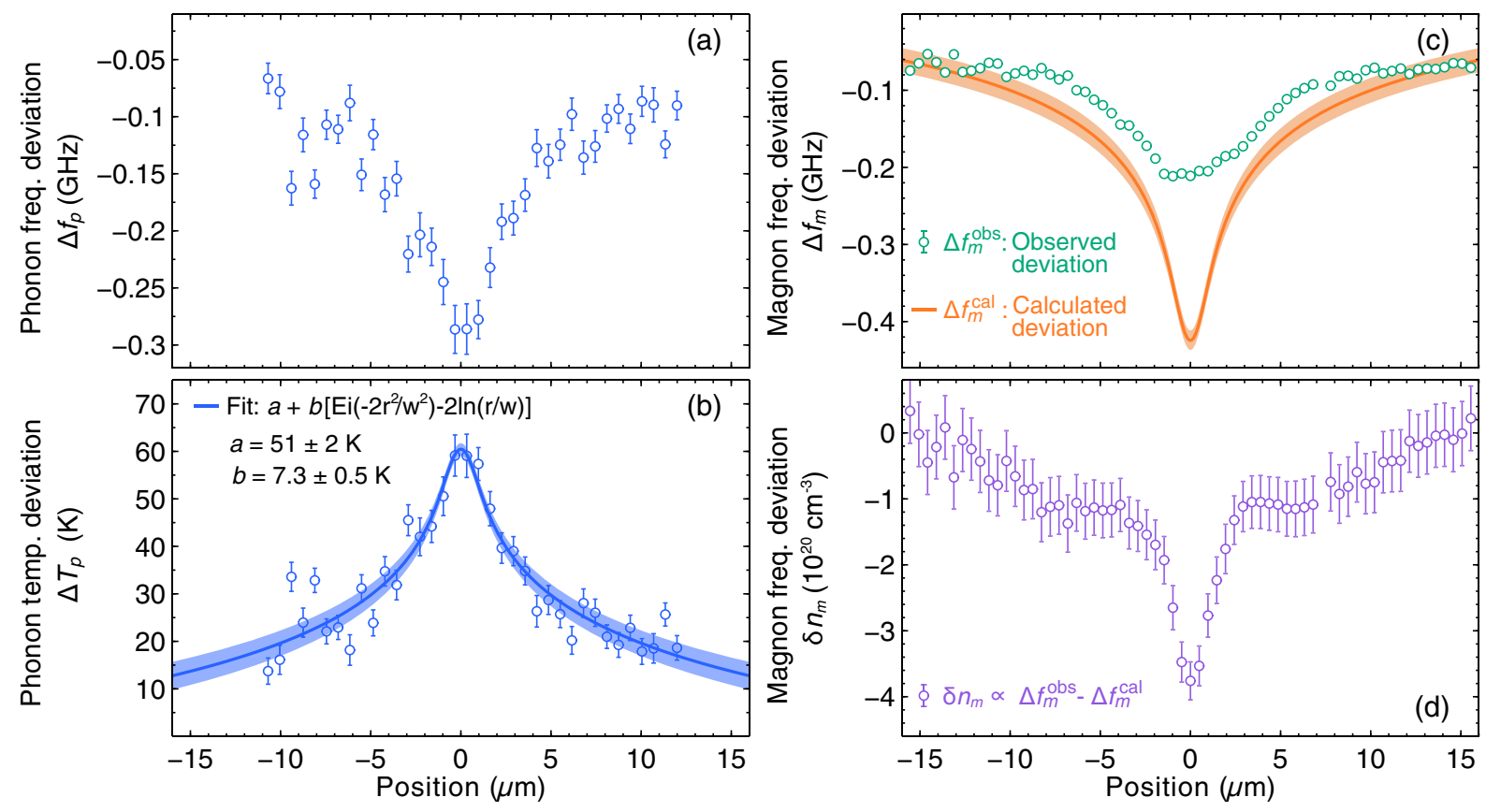

FIG. 3. Spatially resolved phonon temperature and nonequilibrium magnon density. (a) The phonon frequency deviation as a function of the probe laser position, where 0 corresponds to the center of the heating laser. (b) The phonon frequency deviation is converted into an increase of phonon temperature using the frequency-to-temperature calibration (Sec. III C). (c) The observed magnon frequency deviation shown in green circles. Using the temperature-to-magnon-frequency calibration, the calculated magnon deviation (orange line) is based on the simulated magnon temperature profile. (d) The nonequilibrium magnon density $\delta n_{m}$ is proportional to the difference between the calculated and observed magnon frequency deviations. 
function for the phonon temperature profile was approximated as the solution to a simplified heat-transport equation in a 2D axially symmetric geometry (see Supplemental Material [21]).

In quantifying the magnon nonequilibrium, the observed local magnon frequency deviation $\Delta f_{m}^{\text {obs }}$ is compared to the magnon frequency expected with no chemical potential, i.e., the calculated magnon frequency $\Delta f_{m}^{\text {cal }}$. As with the phonon frequency, the observed magnon frequency deviation $\Delta f_{m}^{\text {obs }}=f_{m}^{\text {on }}-f_{m}^{\text {off }}$ measures the magnon frequency change due to the heating laser. Using the magnon temperature calibration function $f_{m}(T)$ (Sec. III C), the equilibrium magnon frequency shift is calculated using local magnon temperature: $\Delta f_{m}^{c a l}=\Delta f_{m}\left(\Delta T_{m}\right)$. This magnon temperature is found through the COMSOL simulation of a magnon-phonon two-temperature model using an energy relaxation length of $\ell_{\epsilon}=250 \mathrm{~nm}$ (see Supplemental Material [21]). Figure 3(c) compares the observed magnon frequency change ( $\Delta f_{m}^{\text {obs }}$ : green circles) to the calculated change from local magnon temperature $\left(\Delta f_{m}^{\text {cal }}\right.$ : red solid line). The clear difference between the two frequencies shows that the magnons are in nonequilibrium under local laser heating.

The magnitude of nonequilibrium magnon density is determined by examining the difference in the observed and equilibrium magnon frequencies: $\delta f_{m}=\Delta f_{m}^{\text {obs }}$ $\Delta f_{m}^{\mathrm{cal}}$. The systematic changes in the local magnon frequency result from changes in the local magnetization $\delta M$, which, in turn, are caused by changes in the magnon number density, i.e., $\delta f_{m} \propto \delta M \propto \delta n_{m}$. Thus, $\delta f_{m}$ measures the frequency deviation resulting from the nonequilibrium magnon density $\delta n_{m}$. The two quantities are related by

$$
\left.\delta f_{m} \approx \frac{\partial f_{m}}{\partial M}\right|_{H} \delta M=-\left.\frac{\partial f_{m}}{\partial M}\right|_{H} g \mu_{B} \delta n_{m},
$$

where $H$ is the constant external field, $g$ is the Lande $g$-factor, and $\mu_{B}$ is the Bohr magneton. The value of $\left.\left(\partial f_{m} / \partial M\right)\right|_{H}$ was obtained from the temperature calibrations (see Supplemental Material [21]) and is used to calculate $\delta n_{m}$ at each position, shown in Fig. 3(d). The measured magnitude of the nonequilibrium magnon density is near $4 \times 10^{20} \mathrm{~cm}^{-3}$ at the maximum. For context, this density, a sum over all thermally excited magnon modes, is about an order of magnitude larger than the nonequilibrium density of a particular magnon mode investigated in parametric pumping experiments $[15,25]$. This broad distribution of the measured magnon nonequilibrium is further characterized by nonequilibrium spectral density, to be discussed in detail later (Sec. IV D).

\section{B. Magnon chemical potential}

For magnons, and bosonlike quasiparticles in general, a nonequilibrium chemical potential can only be appropriately introduced when the quasiparticle number is approximately conserved. Such a quasiequilibrium can be achieved when the quasiparticle interactions occur at a faster rate than the quasiparticle lifetime $[17,26]$. This case allows quasiparticles to redistribute themselves in momentum space before they decay, leading to a distribution with conserved quasiparticle number within the lifetime, i.e., a quasiequilibrium distribution.

In the case of magnons, spin-conserving magnonmagnon interactions redistribute momentum and energy within the magnon system and determines the magnon momentum relaxation rate [10]. On a longer timescale, spin-conserving magnon-phonon interactions relax the magnon energy with the lattice at the energy relaxation rate. Finally, on an even longer timescale, spin nonconserving magnon-magnon and magnon-phonon interactions relax the spin, or magnon number, at the spin relaxation rate. For timescales longer than the magnon momentum relaxation and shorter than the magnon spin relaxation, chemical potential is introduced to describe the magnon distribution.

In steady-state systems, as studied here, relaxation lengths are more relevant. The relations of magnon relaxation times hold similarly for the magnon momentum, energy, and spin relaxation lengths. In YIG, the magnon energy and spin relaxation lengths are about $250 \mathrm{~nm}[12,13]$ and 3-10 $\mu \mathrm{m}$ $[8,9,11]$, respectively. As the magnon momentum relaxation length is shorter than the energy relaxation length, our measurements are within the quasiequilibrium length scale. Thus, a magnon chemical potential $\mu_{m}$ is introduced to the magnon Bose-Einstein (BE) distribution function, and the density of magnons is given by

$$
n_{m}\left(T_{m}, \mu_{m}\right)=\int \frac{d \boldsymbol{k}}{(2 \pi)^{3}} \frac{1}{\exp \left[\frac{\left(\epsilon_{k}-\mu_{m}\right)}{k_{B} T_{m}}\right]-1},
$$

where $T_{m}$ is the magnon temperature and $\epsilon_{k}$ is the magnon energy at wave vector $\boldsymbol{k}$. For further explanation of the magnon chemical potential and quasiequilibrium, see the Supplemental Material [21].

The thermally generated magnon chemical potential can be quantified from spatially resolved nonequilibrium magnon density measurements. The nonequilibrium magnon density is equal to the difference between the total magnon density $n_{m}$ and the equilibrium magnon density $n_{m}^{0}$, respectively, determined from the $\mathrm{BE}$ distribution with and without a chemical potential,

$\delta n_{m}=\int \frac{d \boldsymbol{k}}{(2 \pi)^{3}}\left(\frac{1}{\exp \left[\frac{\left(\epsilon_{k}-\mu_{m}\right)}{k_{B} T_{m}}\right]-1}-\frac{1}{\exp \left[\frac{\epsilon_{k}}{k_{B} T_{p}}\right]-1}\right)$,

where $T_{p}$ is the phonon temperature. The equilibrium magnon density is calculated based on the local lattice (phonon) temperature. Defining the phonon temperature as $T_{p}=T_{m}+\delta T$, where $\delta T$ is the small difference in magnon 

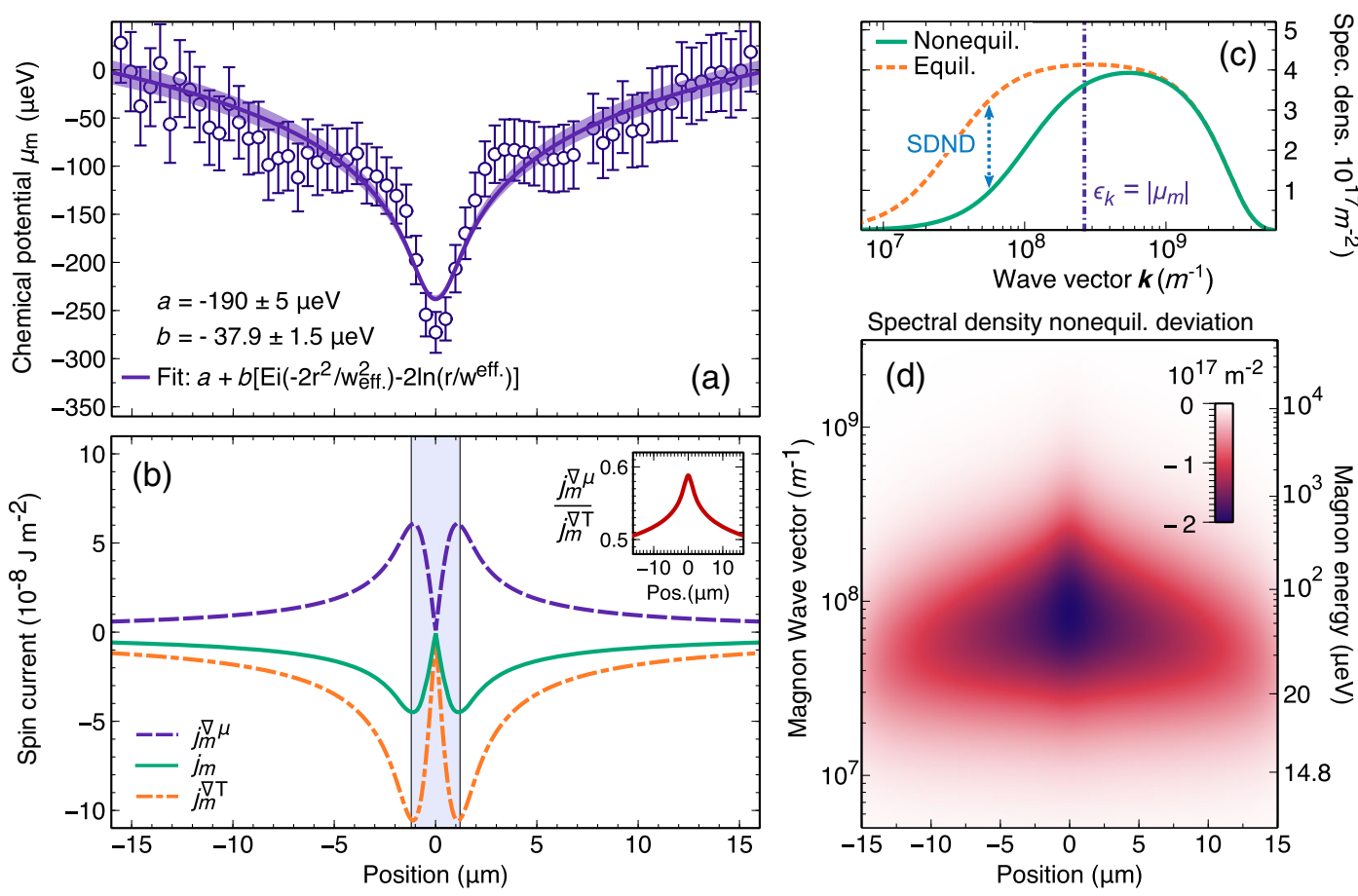

FIG. 4. Magnon chemical potential, spin current, and nonequilibrium spectral density. (a) The magnon chemical potential determined from the local nonequilibrium magnon density, simulated magnon temperature, and the difference between the measured phonon and simulated magnon temperatures. (b) The temperature gradient driven magnon spin current (orange, dot-dashed curve) determined from the simulated magnon temperature, the chemical potential gradient driven spin current (purple, dashed curve) determined from the fit in (a), and the total magnon spin current (green, solid curve) obtained by adding these two spin currents. The inset shows the ratio of the spin current driven by the chemical potential gradient to the total spin currents. The currents flow along the direction of their respective temperature and chemical potential gradients radially. (c) The density of nonequilibrium magnons responsible for carrying the spin current is found from the difference of the total magnon spectral density and the equilibrium spectral densities. These spectral densities are found from the product of the magnon BE distribution function and wave-vector density (see Supplemental Material [21]). The nonequilibrium spectral density (green, solid curve) includes a nonzero magnon chemical potential in the distribution function, whereas the equilibrium spectral density has a chemical potential of zero, the equilibrium value. The blue arrow shows the spectral density nonequilibrium deviation (SDND). (d) The SDND calculated from Eq. (10) using the measured chemical potential and local temperature, which shows the wave vector and energy dependence of the thermally generated nonequilibrium magnons. The total magnon density is reduced from its equilibrium value, hence the negative nonequilibrium density. White represents the lowest magnitude, while purple represents the highest magnitude.

and phonon temperatures, and assuming the chemical potential is small $[15,16,18]$, the nonequilibrium magnon density can be approximated as

$$
\begin{aligned}
\delta n_{m}= & \frac{\mu_{m}}{k_{B} T_{m}} \int \frac{d \boldsymbol{k}}{(2 \pi)^{3}} \frac{\exp \left[\frac{\epsilon_{k}}{k_{B} T_{m}}\right]}{\left(\exp \left[\frac{\epsilon_{k}}{k_{B} T_{m}}\right]-1\right)^{2}} \\
& -\frac{\delta T}{k_{B} T_{m}^{2}} \int \frac{d \boldsymbol{k}}{(2 \pi)^{3}} \frac{\exp \left[\frac{\epsilon_{k}}{k_{B} T_{m}}\right] \epsilon_{\boldsymbol{k}}}{\left(\exp \left[\frac{\epsilon_{k}}{k_{B} T_{m}}\right]-1\right)^{2}} .
\end{aligned}
$$

The chemical potential is found to be linearly related to the nonequilibrium magnon density with a temperature-dependent integration coefficient and an offset that results from the difference in magnon and phonon temperatures. This offset vanishes outside the heating spot as $T_{m}$ approaches $T_{p}$ (see Supplemental Material [21] for details). The values of the chemical potential are shown in Fig. 4(a). For an empirical fitting function, the same phonon temperature function is used, with an effective Gaussian radius $w_{\text {eff }}=1.35 \mu \mathrm{m}$. This empirical fit of the chemical potential is used later in determining the spin current. The peak value of the magnon chemical potential is approximately $-250 \mu \mathrm{eV}$. The negative magnon chemical potential increases the effective magnon energy, thus lowering the total magnon density. This result is consistent with the observation of a reduced magnon frequency deviation [9]. We note that the maximum value of the chemical potential here only changes by less than $2 \%$ when using $T_{m}$ vs $T_{p}$ in Eq. (6) (see Supplemental Material [21]). This result indicates that the degree of magnon nonequilibrium is not determined by the energy relaxation length, which is itself dependent on the magnon-phonon coupling strength. Instead, the degree of magnon nonequilibrium is determined by the spin relaxation length.

\section{Thermally driven spin current}

With the chemical potential quantified, the temperatureand chemical-potential-dependent components of the total 
spin current are independently determined. A single magnon carries $-\hbar$ spin; hence, the spin current $j_{m}$ generated by the nonequilibrium magnons is given by

$$
j_{m}=-\hbar \int \frac{d \boldsymbol{k}}{(2 \pi)^{3}} v_{\boldsymbol{k}}\left[N_{\boldsymbol{k}}\left(T_{m}, \mu_{m}\right)-N_{\boldsymbol{k}}^{0}\left(T_{m}\right)\right],
$$

where $v_{k}=\left(\partial \omega_{k} / \partial k\right)$ is the magnon group velocity, and $N_{k}$ and $N_{k}^{0}$ are the nonequilibrium and equilibrium magnon occupation numbers, respectively. Employing the Boltzmann transport equation and the relaxation time approximation,

$$
\begin{aligned}
N_{k}-N_{k}^{0} & =-\tau_{k} v_{k} \cdot \nabla N_{k} \\
& =-\tau_{k} v_{k} \cdot\left[\frac{\partial N_{k}^{0}}{\partial T} \nabla T_{m}+\frac{\partial N_{k}^{0}}{\partial \mu} \nabla \mu_{m}\right],
\end{aligned}
$$

the spin current can be separated into two components,

$$
\begin{aligned}
& j_{m}^{\nabla T}=\left\{\hbar \int \frac{d \boldsymbol{k} \tau_{\boldsymbol{k}} v_{\boldsymbol{k}}^{2}}{(2 \pi)^{3}} \frac{\exp \left[\frac{\epsilon_{\boldsymbol{k}}}{k_{B} T_{m}}\right]}{\left(\exp \left[\frac{\epsilon_{k}}{k_{B} T_{m}}\right]-1\right)^{2}} \frac{\epsilon_{\boldsymbol{k}}}{k_{B} T_{m}^{2}}\right\} \nabla T_{m}, \\
& j_{m}^{\nabla \mu}=\left\{\hbar \int \frac{d \boldsymbol{k} \tau_{\boldsymbol{k}} v_{\boldsymbol{k}}^{2}}{(2 \pi)^{3}} \frac{\exp \left[\frac{\epsilon_{k}}{k_{B} T_{m}}\right]}{\left(\exp \left[\frac{\epsilon_{k}}{k_{B} T_{m}}\right]-1\right)^{2}} \frac{1}{k_{B} T_{m}}\right\} \nabla \mu_{m},
\end{aligned}
$$

where $\tau_{k}$ is the wave-vector-dependent magnon spin relaxation time [14,27-29]. For the calculation of the spin current, the YIG spin relaxation time is taken from Refs. [14,27], where $\tau_{k}$ follows a second-order polynomial in $k$ and scales linearly with the zero-wave-vector magnon spin relaxation time $\tau_{0}$ (see Supplemental Material [21]). Two components of the spin current are then quantified by integrating Eqs. (8) and (9) and using the gradients of the fitted temperature and chemical potential profiles. Figure 4(b) shows the spin currents driven by the temperature and chemical potential gradient, as well as the total spin current. These currents flow along the radial direction, following the gradients produced by the laser heating.

Spin currents are typically measured via voltage in an adjacent heavy-metal layer [30]. Spin currents measured from the spin Seebeck effect are in the range of $10^{-9}-10^{-8} \mathrm{Jm}^{-2}$ at temperature gradients similar to the one generated by our heating laser $[27,31,32]$. These valus are estimated after making a number of assumptions, including the spin Hall angle, the spin diffusion length in the heavy metal, and interfacial effects. Here, the conversion to spin current depends on the spin relaxation rate $\tau_{k}$ according to Eqs. (8) and (9). The function for $\tau_{k}$ is linearly proportional to the zero-wave-vector magnon spin relaxation time $\tau_{0}$ (see Supplemental Material [21]). A typical value of the Gilbert damping for YIG, $\alpha=$ $2 \times 10^{-4} \quad[8,31,33]$, yields a relaxation time value $\tau_{0}=1.0 \times 10^{-10} \mathrm{~s}$, where $\tau_{0}=\hbar / \alpha k_{B} T[10,34]$. From this $\tau_{0}$, we determine the maximum maganitude of the total spin current, about $5 \times 10^{-8} \mathrm{Jm}^{-2}$, as seen in Fig. 4(b). Though our method for evaluating the spin current in magnetic insulators depends on $\tau_{0}$, which can vary by about 3 to 4 between samples, it is a large improvement over the traditional measurements. Our method removes the ambiguity of interfacial spin-current transmission in a bilayer consisting of a magnetic insulator and a heavy metal. It provides valuable guidance for selecting and optimizing the growth of magnetic insulators for high-efficiency generation of pure spin current.

Next, we examine the ratio between the chemicalpotential- and temperature-gradient-driven spin currents, shown in the inset of Fig. 4(b). The ratio peaks as it approaches the center, with a maximum of approximately $60 \%$, and then decreases outward. This ratio is independent of the zero-wave-vector magnon spin relaxation time $\tau_{0}$. Thus, we conclude that the chemical potential associated with the spin current significantly contributes to the total magnon spin current.

\section{Magnon nonequilibrium spectral density}

Finally, the energy and wavelength range of magnons that contribute to the spin current has long been debated since the initial theories of the spin Seebeck effect were developed $[35,36]$. Previous studies have suggested that magnon nonequilibrium is energy dependent, and low-energy magnons ( $3 \mathrm{meV}$ and below), dubbed subthermal magnons, dominantly contribute to the spin Seebeck effect [37]. The energy dependence of the nonequilibrium magnon density is determined by examining the magnon spectral density nonequilibrium deviation (SDND),

$$
\delta \rho(k)=\frac{k^{2} / 2 \pi^{2}}{\exp \left[\frac{\left.\epsilon_{k}-\mu_{m}\right)}{k_{B} T_{m}}\right]-1}-\frac{k^{2} / 2 \pi^{2}}{\exp \left[\frac{\epsilon_{k}}{k_{B} T_{p}}\right]-1} .
$$

The SDND [Fig. 4(c)] is calculated following Eq. (10). This calculation includes the values of the chemical potential from Fig. 4(a), the local magnon and phonon temperatures, and the magnon dispersion $\epsilon_{\mathbf{k}}=\hbar \omega_{k}$ (see Sec. III B). A maximum occurs for magnons of wave vectors in the range $(0.4-2.0) \times 10^{8} \mathrm{~m}^{-1}$, corresponding to energies 30-200 $\mu \mathrm{eV}$ and wavelengths of about 30$150 \mathrm{~nm}$. This energy range results from a combination of the chemical potential energy and density of states. The energy width of the SDND approximately equals the magnitude of the chemical potential. While our study also supports the qualitative conclusion that the highest magnon nonequilibrium occurs in low-energy magnons, the energies shown here are 1 to 2 orders of magnitude lower than the value quoted from previous studies [37]. The spectral distribution of the nonequilibrium magnons shown in Fig. 4(c) demonstrates that the magnon spin current is 
carried by the magnons within a rather narrow energy and wave vector range. This result has implications for spinbased devices that exploit a thermal gradient.

\section{CONCLUSIONS}

In summary, by exploring BLS as an experimental method that directly probes the nonequilibrium magnon density, we extract the spatially varying chemical potential and quantitatively evaluate the spin current in a magnetic insulator subject to a thermally driven nonequilibrium. There are several valuable insights to be drawn from these results. First, interfacial spin transmission effects, omnipresent in transport measurements, are completely removed in this method. One can evaluate magnetic insulators on their intrinsic spin-current generation capability. Second, the analysis of the magnon spectral densities reveals how exactly the nonequilibrium is distributed over magnons of different energy and wavelength. Device parameters, such as film thickness and antenna width, can be designed with such information in mind to enhance the nonequilibrium magnon density and spin currents. Third, our findings show that assuming equal magnon and phonon temperatures or using simulated magnon temperatures has had a limited influence on the spin current. Accordingly, we suggest that magnon spin relaxation, instead of magnon-phonon energy relaxation strength, plays a more important role in spincurrent generation. Finally, our results demonstrate that the concept of magnon chemical potential can successfully describe a general nonequilibrium magnet, thus laying the groundwork for a better understanding of broad nonequilibrium phenomena.

\section{ACKNOWLEDGMENTS}

This research (X.L, G.F. and J.Z.) was primarily supported by the National Science Foundation through the Center for Dynamics and Control of Materials: an NSF MRSEC under Cooperative Agreement No. DMR1720595. K. S. O. and X. L. were partially supported as part of SHINES, an Energy Frontier Research Center funded by the U.S. Department of Energy (DOE), Office of Science, Basic Energy Science (BES) under Award No. DE-SC0012670. G. F. and J.Z. acknowledge partial funding by the NSF via Grant No. DMR-1949701. K. An acknowledges support from the European Union's Horizon 2020 research and innovation programme under the Marie Sklodowska-Curie Grant agreement No. 665667. J. Z. and L. S. were supported by the Army Research Office (ARO) MURI program under Grant No. W911NF-14-1-0016.

K. S. O. and K. A. acquired data. K. S. O., L. S., and G. F. performed analysis and theoretical studies. J. Z. provided the YIG sample. K.S.O. and X.L. wrote the original manuscript. L. S., J.Z., and X. L. supervised the project. All authors assisted in the discussion of results and revision of the manuscript
[1] V. V Kruglyak, S. O. Demokritov, and D. Grundler, Magnonics, J. Phys. D 43, 264001 (2010).

[2] A. A. Serga, A. V. Chumak, and B. Hillebrands, YIG Magnonics, J. Phys. D 43, 264002 (2010).

[3] J. Sinova and I. Žutić, New Moves of the Spintronics Tango, Nat. Mater. 11, 368 (2012).

[4] G. E. W. Bauer, E. Saitoh, and B. J. Van Wees, Spin Caloritronics, Nat. Mater. 11, 391 (2012).

[5] S. R. Boona, R. C. Myers, and J. P. Heremans, Spin Caloritronics, Energy Environ. Sci. 7, 885 (2014).

[6] H. Yu, S. D. Brechet, and J. P. Ansermet, Spin Caloritronics, Origin and Outlook, Phys. Lett. A 381, 825 (2017).

[7] S. S. L. Zhang and S. Zhang, Magnon Mediated Electric Current Drag across a Ferromagnetic Insulator Layer, Phys. Rev. Lett. 109, 096603 (2012).

[8] L. J. Cornelissen, J. Liu, R. A. Duine, J. B. Youssef, and B. J. Van Wees, Long-Distance Transport of Magnon Spin Information in a Magnetic Insulator at Room Temperature, Nat. Phys. 11, 1022 (2015).

[9] K. An, K. S. Olsson, A. Weathers, S. Sullivan, X. Chen, X. Li, L. G. Marshall, X. Ma, N. Klimovich, J. Zhou, L. Shi, and X. Li, Magnons and Phonons Optically Driven out of Local Equilibrium in a Magnetic Insulator, Phys. Rev. Lett. 117, 107202 (2016).

[10] L. J. Cornelissen, K. J. H. Peters, G. E. W. Bauer, R. A. Duine, and B. J. Van Wees, Magnon Spin Transport Driven by the Magnon Chemical Potential in a Magnetic Insulator, Phys. Rev. B 94, 014412 (2016).

[11] B. L. Giles, Z. Yang, J. S. Jamison, J. M. Gomez-Perez, S. Vélez, L. E. Hueso, F. Casanova, and R. C. Myers, Thermally Driven Long-Range Magnon Spin Currents in Yttrium Iron Garnet Due to Intrinsic Spin Seebeck Effect, Phys. Rev. B 96, 180412(R) (2017).

[12] A. Prakash, B. Flebus, J. Brangham, F. Yang, Y. Tserkovnyak, and J.P. Heremans, Evidence for the Role of the Magnon Energy Relaxation Length in the Spin Seebeck Effect, Phys. Rev. B 97, 020408(R) (2018).

[13] M. Agrawal, V. I. Vasyuchka, A. A. Serga, A. Kirihara, P. Pirro, T. Langner, M. B. Jungfleisch, A. V. Chumak, E. T. Papaioannou, and B. Hillebrands, Role of BulkMagnon Transport in the Temporal Evolution of the Longitudinal Spin-Seebeck Effect, Phys. Rev. B 89, 224414 (2014).

[14] S. M. Rezende, A. Azevedo, and R. L. Rodríguez-Suárez, Magnon Diffusion Theory for the Spin Seebeck Effect in Ferromagnetic and Antiferromagnetic Insulators, J. Phys. D 51, 174004 (2018).

[15] S. O. Demokritov, V. E. Demidov, O. Dzyapko, G. A. Melkov, A. A. Serga, B. Hillebrands, and A. N. Slavin, Bose-Einstein Condensation of Quasi-Equilibrium Magnons at Room Temperature under Pumping, Nature (London) 443, 430 (2006).

[16] C. Du, T. van der Sar, T. X. Zhou, P. Upadhyaya, F. Casola, H. Zhang, M. C. Onbasli, C. A. Ross, R. L. Walsworth, Y. Tserkovnyak, and A. Yacoby, Control and Local Measurement of the Spin Chemical Potential in a Magnetic Insulator, Science 357, 195 (2017).

[17] L. H. Bennett and E. Della Torre, The Chemical Potential of Magnons in Quasi-Equilibrium, Phys. B Condens. Matter 403, 324 (2008). 
[18] V. E. Demidov, S. Urazhdin, B. Divinskiy, V. D. Bessonov, A. B. Rinkevich, V. V. Ustinov, and S. O. Demokritov, Chemical Potential of Quasi-Equilibrium Magnon Gas Driven by Pure Spin Current, Nat. Commun. 8, 1579 (2017).

[19] K. S. Olsson, N. Klimovich, K. An, S. Sullivan, A. Weathers, L. Shi, and X. Li, Temperature Dependence of Brillouin Light Scattering Spectra of Acoustic Phonons in Silicon, Appl. Phys. Lett. 106, 051906 (2015).

[20] K. S. Olsson, K. An, X. Ma, S. Sullivan, V. Venu, M. Tsoi, J. Zhou, L. Shi, and X. Li, Temperature-Dependent Brillouin Light Scattering Spectra of Magnons in Yttrium Iron Garnet and Permalloy, Phys. Rev. B 96, 024448 (2017).

[21] See Supplemental Material at http://link.aps.org/ supplemental/10.1103/PhysRevX.10.021029 for more details on the calibration procedure, heating effects, temperature profile simulations, theory of the magnon chemical potential, and spin current derivations.

[22] S. Kimura and I. Shindo, Single Crystal Growth of YIG by the Floating Zone Method, J. Cryst. Growth 41, 192 (1977).

[23] E. J. J. Mallmann, A. S. B. Sombra, J. C. Goes, and P. B. A. Fechine, Yttrium Iron Garnet: Properties and Applications Review, Solid State Phenom. 202, 65 (2013).

[24] H. Suhl, The Theory of Ferromagnetic Resonance at High Signal Powers, J. Phys. Chem. Solids 1, 209 (1957).

[25] A. A. Serga, T. Neumann, and B. Hillebrands, Probing of a Parametrically Driven Magnon Gas with a Non-resonant Traveling Spin Waves, Appl. Phys. Lett. 93, 252501 (2008).

[26] D. Snoke, Coherent Questions, Nature (London) 443, 403 (2006).

[27] S. M. Rezende, R. L. Rodríguez-Suárez, R. O. Cunha, J. C. L. Ortiz, and A. Azevedo, Bulk Magnon Spin Current Theory for the Longitudinal Spin Seebeck Effect, J. Magn. Magn. Mater. 400, 171 (2016).
[28] S. M. Rezende, R. L. Rodríguez-Suárez, and A. Azevedo, Diffusive Magnonic Spin Transport in Antiferromagnetic Insulators, Phys. Rev. B 93, 054412 (2016).

[29] G. Chen, Nanoscale Energy Transport and Conversion: A Parallel Treatment of Electrons, Molecules, Phonons, and Photons (Oxford University, New York, 2005).

[30] S. Maekawa, H. Adachi, K. Uchida, J. Ieda, and E. Saitoh, Spin Current: Experimental and Theoretical Aspects, J. Phys. Soc. Japan, 82, 102002 (2013).

[31] H. Chang, P. A. Praveen Janantha, J. Ding, T. Liu, K. Cline, J. N. Gelfand, W. Li, M. C. Marconi, and M. Wu, Role of Damping in Spin Seebeck Effect in Yttrium Iron Garnet Thin Films, Sci. Adv. 3, e1601614 (2017).

[32] J. Holanda, O. A. Santos, R. L. Rodríguez-Suárez, A. Azevedo, and S. M. Rezende, Simultaneous Spin Pumping and Spin Seebeck Experiments with Thermal Control of the Magnetic Damping in Bilayers of Yttrium Iron Garnet and Heavy Metals: YIG/Pt and YIG/IrMn, Phys. Rev. B 95, 134432 (2017).

[33] L. Jin, Y. Wang, G. Lu, J. Li, Y. He, Z. Zhong, and H. Zhang, Temperature Dependence of Spin-Wave Modes and Gilbert Damping in Lanthanum-Doped Yttrium-IronGarnet Films, AIP Adv. 9, 025301 (2019).

[34] M. Schreier, A. Kamra, M. Weiler, J. Xiao, G. E. W. Bauer, R. Gross, and S. T. B. Goennenwein, Magnon, Phonon, and Electron Temperature Profiles and the Spin Seebeck Effect in Magnetic Insulator/Normal Metal Hybrid Structures, Phys. Rev. B 88, 094410 (2013).

[35] J. Xiao, G. E. W. Bauer, K. C. Uchida, E. Saitoh, and S. Maekawa, Theory of Magnon-Driven Spin Seebeck Effect, Phys. Rev. B 81, 214418 (2010).

[36] S. Hoffman, K. Sato, and Y. Tserkovnyak, Landau-Lifshitz. Theory of the Longitudinal Spin Seebeck Effect, Phys. Rev. B 88, 064408(2013).

[37] T. Kikkawa, K. I. Uchida, S. Daimon, Z. Qiu, Y. Shiomi, and E. Saitoh, Critical Suppression of Spin Seebeck Effect by Magnetic Fields, Phys. Rev. B 92, 064413 (2015). 